(where the superscript is reduced, modulo $n_{i}$, if necessary) be a homeomorphism agreeing with $T$ on $L$ and sending $b_{i}^{j}$ into $b_{i}^{j+1}$ (with the same convention on the superscripts). This defines $T$ for every $p$ of $M$. It is evident that $T(M)=M$ is a pointwise periodic homeomorphism.

If we now define

$$
G_{i}=\sum_{j=1}^{n_{i}} b_{i}^{j}
$$

we see that each $G_{i}$ is an orbit under $T$, and conditions (a) and (b) of the theorem are satisfied. The proof is thus complete.

University of PENNSYlvania AND

UNIVERSITY OF VIRGINIA

\title{
AN ENUMERATION OF LOGICAL FUNCTIONS
}

\section{WILLIAM WERNICK}

In a logical calculus of $m$ values, abbreviated by $L_{m}$, we may deal with functions of $n$ variables. A particular function is defined in this calculus if we assign a constant value, which may be any arbitrary one of the $m$ possible values in $L_{m}$, as the value of that function for a particular argument. It is the purpose of this note to enumerate, among all functions of $n$ variables in $L_{m}$ : those which depend on all $n$ variables in the argument; those which depend on just $(n-1)$ of the variables in the argument, being independent of one of them; and so on; finally those which are completely independent of all the variables in the argument.

Since each variable in the argument may assume values from $1, \cdots, m$, independently, there are $m^{n}$ possible arguments, and since to each argument we may assign independently, as a functional value, any of the $m$ values $1, \cdots, m$, there are in all $m^{m^{n}}$ possible functions of $n$ variables.

Let $V_{n}$ be the total number of all functions of $n$ variables in $L_{m}$. Then we have from the above

$$
V_{n}=m^{m^{n}} .
$$

Let $U_{n k}$ be the number of functions of $n$ variables which depend on exactly $k$ of them. (It is this expression for which we are seeking an explicit evaluation.) Since $k$ variables may be selected from $n$ of them in just $C_{n, k}$ ways, we have the relation:

$$
U_{n k}=C_{n, k} U_{k k} .
$$


Since $V_{n}$ includes all functions of $n$ variables in $L_{m}$, we may write $V_{n}$ as the sum of: all functions of $n$ variables which depend on all of them; all functions of $n$ variables which depend on all but one of them; and so on; that is, $V_{n}=\sum_{k=0}^{n} U_{n k}$, which, by the relation above, becomes

$$
V_{n}=\sum_{k=0}^{n} C_{n, k} U_{k k}
$$

This leads to the following implicit recursive definition for $U_{k k}$ :

$$
\begin{aligned}
V_{0} & =U_{00}, \\
V_{1} & =C_{1,0} U_{00}+C_{1,1} U_{11}, \\
V_{2} & =C_{2,0} U_{00}+C_{2,1} U_{11}+C_{2,2} U_{22}, \\
\text {. . . . . . . . . . . . . . . } & . \\
V_{k} & =C_{k, 0} U_{00}+C_{k, 1} U_{11}+\cdots+C_{k, k} U_{k k} .
\end{aligned}
$$

We may solve this system of $k+1$ equations in the $k+1$ unknowns $U_{00}, U_{11}, \cdots, U_{k k}$ by the use of Cramer's rule and some manipulation of determinants to obtain the value for $U_{k k}:^{*}$

$$
U_{k k}=\sum_{i=0}^{k}(-1)^{i} C_{k, i} V_{k-i},
$$

which by application of (1) gives the explicit value we want for $U_{k k}$ :

$$
U_{k k}=\sum_{i=0}^{k}(-1)^{i} C_{k, i} m^{m^{k-i}} .
$$

Finally, using this result in formula (2), we have the actual enumeration formula; that is, the number of functions of $n$ variables in $L_{m}$ which depend on exactly $k$ of them is given by the formula:

$$
U_{n k}=C_{n, k} \sum_{i=0}^{k}(-1)^{i} C_{k, i} m^{m^{k-i}} .
$$

REMARK. We may, in formula (4), make use of the apparent analogy with the binomial expansion. If we agree to interpret, that is, symbolize, $V_{i}$ as $v^{i}$, where the $i$ in $v^{i}$ is an exponent, and write this interpretation $V_{i} \equiv v^{i}$, we have, for example, $V_{0} \equiv v_{0}=1$. $\dagger$ Then our

* I am indebted for the actual carrying out of this solution to Dr. J. C. C. McKinsey. The solution, though elementary, is quite lengthy and may be left as an exercise for the reader.

$\dagger$ We write $\equiv$ to mean "symbolically equals," and = to mean "equals." These are not interchangeable, since $V_{0}$ is not equal to 1 , but to $m$. 
equation (4) becomes

$$
U_{k k} \equiv \sum_{i=0}^{k} C_{k, i}(-1)^{i} v^{k-i} .
$$

But this expansion on the right is simply the binomial expansion for $(v-1)^{k}$, so our simplified formula now is

$$
U_{k k} \equiv(v-1)^{k} \text {. }
$$

For the term $U_{00}$ we get $U_{00} \equiv(v-1)^{0}=1=v^{0} \equiv V_{0}$ as required in $\left(4_{0}\right)$. We get corresponding definitions for the others:

$$
\begin{aligned}
& U_{11}^{\prime}=V_{1}-V_{0}, \\
& U_{22}=V_{2}-2 V_{1}+V_{0},
\end{aligned}
$$

Each of these is made explicit by the use of formula (1). The simplified formula for $U_{n k}$ is now

$$
U_{n k} \equiv C_{n, k}(v-1)^{k} .
$$

To evaluate a particular term, expand the right side, and apply the definitions: $v^{0} \equiv V_{0}=m^{m^{0}}, v^{i} \equiv V_{i}=m^{m^{i}}$.

Application. If $L_{m}$ is the two valued calculus of sentences, and if we consider truth functions of two variables $f_{j}(p, q)$, we have $m=2$ and $n=2$. Then $V_{2}=2^{2^{2}}=16$. There are 16 possible truth functions of two variables in $L_{m}$. Of these 16 functions, there are $U_{20}=C_{2,0} 2^{2^{0}}=2$ functions that depend on 0 variables, that is, that are independent of both $p$ and $q$ (" $f(p, q)$ is always true," " $g(p, q)$ is always false"). There are $U_{21}=C_{2,1}\left(2^{2^{1}}-2^{2^{0}}\right)=4$ functions that depend on one variable only, that is, on $p$ alone or on $q$ alone, but not on both (" $p$ is true," " $p$ is false," " $q$ is true," and " $q$ is false"). There are $U_{22}=C_{2,2}\left(2^{2^{2}}-2 \cdot 2^{2^{1}}+2^{2^{0}}\right)=10$ functions that depend on both variables (all the remaining functions in $V_{2}$, including conjunction, disjunction, implication, and so on).

New York UnIVERSITY 